\title{
Effects of Particle Hydrophilicity and Morphology on Aerosol Scavenging in a Wet Electrostatic Scrubber
}

\author{
Lipeng Su${ }^{1,2}$, Qian Du ${ }^{1 *}$, Min Tang ${ }^{2 *}$, Qisheng $\mathrm{Ou}^{2}$, David Y.H. Pui ${ }^{2,3}$ \\ ${ }^{1}$ School of Energy Science and Engineering, Harbin Institute of Technology, Harbin, Heilongiang 150001, China \\ ${ }^{2}$ Particle Technology Laboratory, University of Minnesota, Minneapolis, MN 55455, USA \\ ${ }^{3}$ The Chinese University of Hong Kong, Shenzhen, Guangdong 518172, China
}

\begin{abstract}
In this work, influences of particle hydrophilicity and morphology on its removal by electrically neutral and positively charged water droplets were investigated experimentally in a home-made chamber. Potassium chloride (KCl) and Di-EthylHexyl-Sebacate (DEHS) and agglomerated $\mathrm{KCl}$ aerosols were successively adopted as representative testing cases and each test was carried out batchwise. The results showed that minimal collection efficiency of DEHS particles (hydrophobic) could be improved from $38 \%$ to $82 \%$ due to droplet charging after spray for ten minutes, which is a significantly greater increment than that of $\mathrm{KCl}$ particles (hydrophilic) after same charging-spray time. Additionally, droplet charging enhances the operational stability of traditional spray to collect DEHS particles in tested sizes while contributes most to removal of $\mathrm{KCl}$ particles only in low concentration and intermediate size. As a comparison with nearly spherical particles (KCl), a higher collection efficiency by electrically neutral droplets corresponds to loose agglomerates in low particle concentration due to interception mechanism, but the difference could be weakened by an addition of electric field onto water spray.
\end{abstract}

Keywords: Hydrophilicity; Particle morphology; Droplet charging.

\section{INTRODUCTION}

Over past few years, air pollution mainly caused by $\mathrm{PM}_{2.5}$ was commonly recognized as the one of most intractable issues in China to improve human settlement environment (Li et al., 2015; Zheng et al., 2016). Numerous studies showed that the $\mathrm{PM}_{2.5}$ concentration of the majority area in China severely exceeded the standard promulgated by the U.S. Environmental Protection Agency (U.S. EPA) (Song et al., 2017; Zhang et al., 2017). Exposure to $\mathrm{PM}_{2.5}$ at such high concentration for a long time may trigger some kinds of serious diseases, such as cardiovascular and respiratory diseases, nervous system and sex-biased neurobehavioral disorders (Genc et al., 2012; Becerra et al., 2013; Sobolewski et al., 2018).

In order to mitigate the $\mathrm{PM}_{2.5}$ pollution in China, a great deal of strategies and technologies emerged constantly, mainly involving high-efficient filters (Chang et al., 2016; Chen et al., 2016; Choi et al., 2017; Zhang et al., 2017), electrostatic precipitators (Park et al., 2017; Liao et al., 2018), wet removal (Jaworek et al., 2006a) and hybrid system

\footnotetext{
${ }^{*}$ Corresponding author.

E-mail address: duqian@hit.edu.cn (Q. Du); tangminde@163.com (M. Tang)
}

(Han et al., 2018; Jaworek et al., 2018). Besides, other new concepts were proposed by some scholars to manage $\mathrm{PM}_{2.5}$ pollution in megacities of China (Cao et al., 2018). Yu et al. (2014) proposed a geoengineering approach for reducing air pollution in megacities by means of water spray into atmosphere like watering garden. Zhou et al. (2015) introduced a Solar Chimney configuration which can drive the warm airstream from ground to high altitude due to the effect of natural buoyancy, but no filters were assembled to mitigate $\mathrm{PM}_{2.5}$ in this system. Based on similar ideas, Cao et al. (2015) showed a Solar-Assisted Large-Scale Cleaning System (SALSCS) to remediate urban $\mathrm{PM}_{2.5}$ by coupling High efficiency particle air (HEPA) filters. However, HEPA filters significantly increased the resistance of airflow rising by solar energy in tower, hence the new generational SALSCS equipped with charging water spray was conceived to resolve this issue and maintain high purification efficiency.

Charging water spray was widely perceived as a reliable technique to achieve high purification efficiency of submicron particles with low pressure drop (Pilat et al., 1974; Metzler et al., 1997; Balachandran et al., 2003; Jaworek et al., 2006b; Carotenuto et al., 2010; D'Addio et al., 2013; Jaworek et al., 2013; D'Addio et al., 2014; Kim et al., 2014; Di Natale et al., 2015) Balachandran et al. (2003) investigated experimentally the suppression rate of cigarette smoke by charged droplets in a closed chamber, revealing that charged droplets could provide two times removal efficiency higher 
than uncharged droplets. Ha et al. (2010) evaluated the scavenging rate of diesel particulate matter collected by charging droplets, which is directly related to water electrical properties, spraying performance and corona power. D'Addio et al. $(2013,2014)$ designed a lab-scale WES (Wet electrostatic scrubbing) system to decouple the influences of droplet-particle interactions and study quantitatively the role of charged droplets in removing submicron particles. Di Natale et al. $(2015,2016,2018)$ performed a series of WES experiments, confirming its good effectiveness to remove submicron particles including gasoline and bacterial particles. Additionally, Jaworek et al. (2002) numerically simulated and reviewed the scavenging rate of small particles by charged droplets, suggesting that smaller droplet size, lower Stokes number and higher Coulomb number have positive effects on collection efficiency. Zhao and Zheng (2008) proposed a population balance model to optimize operational parameters of WES device, indicating that slower droplet velocity, larger droplet charge-to-mass ratio and liquid-to-gas flow ratio are beneficial to the particle collection efficiency. Carotenuto et al. (2010) developed a mathematical model which revealed the droplet-gas relative velocity plays different roles for submicron and microparticles. Even though many results were reported to reveal the influence factors of WES efficiency, there was few studies to expatiate the effects of particle properties to best of our knowledge.

In this study, effects of particle hydrophilicity and morphology on aerosol scavenging were investigated in a home-made electrostatic scrubber. Two kinds of aerosol particles, potassium chloride $(\mathrm{KCl})$ and Di-Ethyl-HexylSebacate (DEHS) were first adopted to elaborate the influence of particle hydrophilicity on its removal. In order to accomplish this detailed measurement in a sufficiently wide size range, a Scanning Mobility Particle Sizer (SMPS) and an Aerodynamic Particle Sizer (APS) were employed in this study. Secondly, nearly spherical and agglomerated
$\mathrm{KCl}$ aerosols were used as representative cases, which aims at expatiating the effect of particle morphology on scrubbing performance. Experiment results showed that both particle hydrophilicity and morphology have significant influences on its removal by electrically neutral or positively charged droplets.

\section{METHODOLOGY}

\section{Setup and Protocol}

The layout of experimental apparatus used in this study is shown in Fig. 1. It mainly consists of a wet electrostatic scrubber chamber, spray droplet charging section, particle generation section and measurement system. The scrubbing chamber was made of transparency glass and designed as a regular hexagon with side and height that are approximately $0.3 \mathrm{~m}$ and $1.1 \mathrm{~m}$, respectively. On the side surfaces of chamber, two air-vents were designed to guarantee the pressure balance in chamber by particle-free air passing through HEPA filters only. At the top cap of chamber, four aerosol inlets were arranged to evenly disperse particles into the chamber. Polydisperse aerosol used in this study was generated from a collision-type atomizer (TSI 3079) which was followed by a diffusion dryer filled with silica gel or active carbon to dry $\mathrm{KCl}$ and DEHS aerosol, respectively. Hydrophobic DEHS particles (oil droplets) are spherical and hydrophilic $\mathrm{KCl}$ particles are nearly spherical, which were tested by Transmission Electron Microscope (TEM) in this study. After diffusion dryer, aerosol particles then pass through a neutralizer to yield overall charge neutral aerosol and flow into the chamber subsequently from top inlets.

Besides aerosol inlets, a pressure nozzle (ID $=1 \mathrm{~mm}$ ) was located in the center of the top cap and connected to the ground as a zero-potential electrode. At the bottom of this nozzle, a copper ring $(\mathrm{OD}=28 \mathrm{~mm}, \mathrm{ID}=23 \mathrm{~mm})$ was fixed at the same horizontal plane as the tip of nozzle and applied a negative potential by a high voltage power supply.

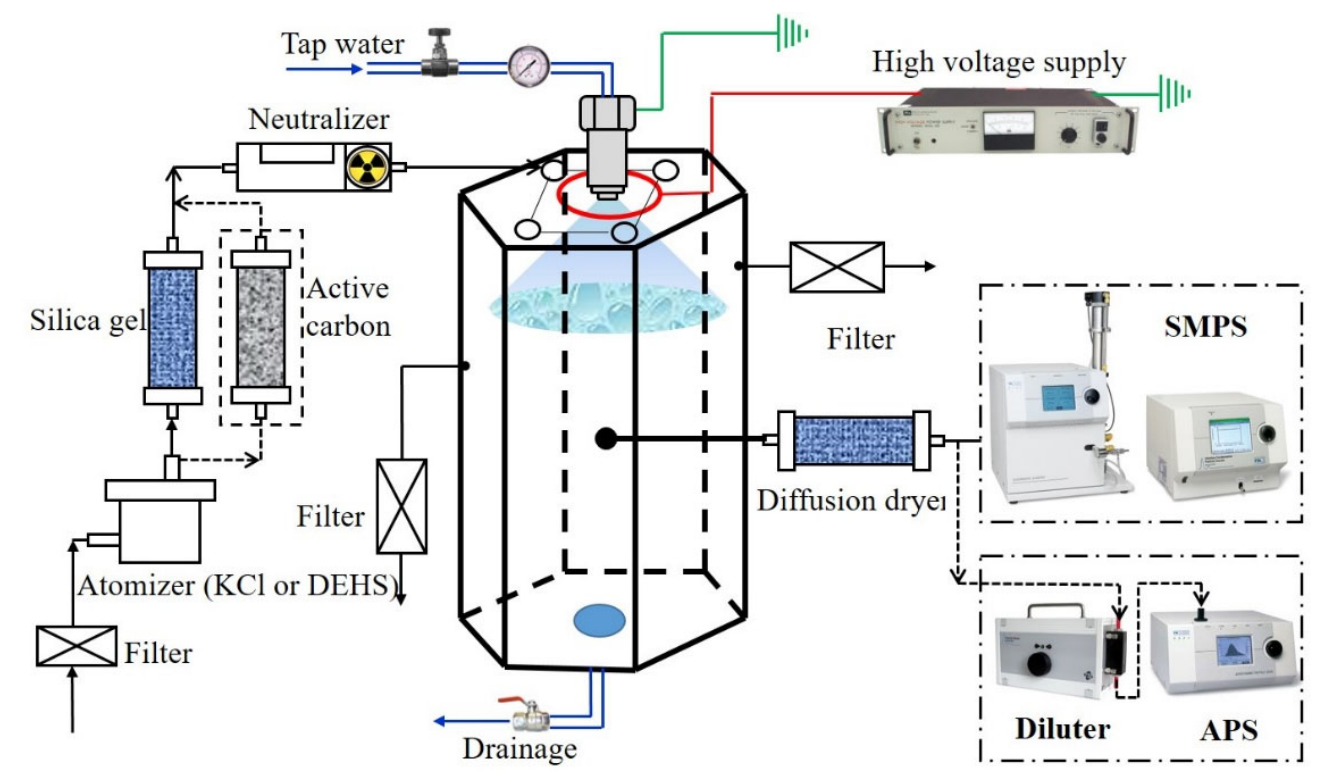

Fig. 1. Schematic diagram of experimental setup used in this study. 
Droplets produced by the nozzle, as a conical spray coaxial to the ring, would pass through the grounded ring, thereby producing net positive charges on the surface of droplets by induction charging mechanism. Without charging, the mean size of spray droplets is approximately estimated from $80 \mu \mathrm{m}$ to $110 \mu \mathrm{m}$ according to the data from nozzle manufacturing company and Ha's study (Ha et al., 2010), while the effect of droplet charging on its mean size is not taken into account in this study due to low electric potential (Ha et al., 2010). Droplet charges acquired by induction charging could be calculated by the following Eq. (1) (Mazumder et al., 2006):

$q_{d}=4 \pi \varepsilon_{0}\left(1.64 E_{0} r_{d}^{2}\right)$

where $\varepsilon_{0}$ is permittivity of free space, $E_{0}$ is average electric field and $r_{d}$ droplet size. Corresponding charges estimated by Eq. (1) is shown in Table 1.

In the whole experiment, a SMPS (3080, TSI) was used to continuously monitor particle concentration and size distribution by classification and scanning modes, respectively. The sampling location was set in the center of chamber to minimize the effect of particle spatial distribution in spray tower. In order to reduce the effect of moisture on measurement, a diffusion dryer was added into the sampling system regardless of spraying. Besides SMPS, a diluter (dilution ratio of 100) and a APS (3321, TSI) were also employed to monitor the large DEHS particles which SMPS is incapable to detect. Even though the APS adopts a different equivalent particle diameter from SMPS, it could also be used to analyze DEHS removal comprehensively.

\section{Agglomerates Generation Method}

In order to expatiate the effect of particle morphology on spray scavenging, a composite aerosol production mode was designed to generate polydisperse $\mathrm{KCl}$ agglomerates, as shown in Fig. 2. A collision-type atomizer (TSI 3076) filled with $0.01 \% \mathrm{KCl}$ aqueous solution was adopted first to generate nanoparticles in a high concentration. These nanoparticles then pass through a diffusion dryer to maintain a low humidity in aerosol flow and particles after dehumidification are introduced into a tube furnace $\left(1100^{\circ} \mathrm{C}\right)$. In this furnace, nanoparticles undergo a full and rapid gasification process to yield $\mathrm{KCl}$ vapor in a high concentration. Subsequently, $\mathrm{KCl}$ vapor coming out of furnace rapidly condenses into a large number of primary particles of which typical size is ten to twenty nanometers. These primary particles then pass through two tandem agglomeration chambers and a Po-210 neutralizer to generate loose and electrically neutral agglomerates. In order to validate particle morphology generated by this method, a TEM test was also conducted and corresponding image was compared with nearly spherical $\mathrm{KCl}$ particle produced by collision-type atomizer directly.

\section{RESULTS AND DISCUSSION}

In this section, $\mathrm{KCl}$ particles, as hydrophilic particles, were investigated first to acquire the particle scavenging process in detail by charged droplets. Then collection performance of hydrophobic particles (DEHS) in chamber was analyzed by aid of SMPS and APS. On this basis, overall comparisons between $\mathrm{KCl}$ and DEHS particles were presented and analyzed to elaborate the effect of particle wettability. Finally, fractal-like $\mathrm{KCl}$ agglomerates, as a comparison to nearly spherical particles, was adopted to expatiate the influence of particle shape on scavenging efficiency.

Table 1. Droplet charges acquired by induction charging.

\begin{tabular}{llll}
\hline$D_{d}(\mu \mathrm{m})$ & 80 & 95 & 110 \\
\hline$q_{d}(\mathrm{C}, U=1 \mathrm{kV})$ & $2.08 \times 10^{-14}$ & $2.93 \times 10^{-14}$ & $3.94 \times 10^{-14}$ \\
$q_{d}(\mathrm{C}, U=2 \mathrm{kV})$ & $4.17 \times 10^{-14}$ & $5.87 \times 10^{-14}$ & $7.88 \times 10^{-14}$ \\
$q_{d}(\mathrm{C}, U=3 \mathrm{kV})$ & $6.25 \times 10^{-14}$ & $8.81 \times 10^{-14}$ & $1.18 \times 10^{-13}$ \\
\hline
\end{tabular}

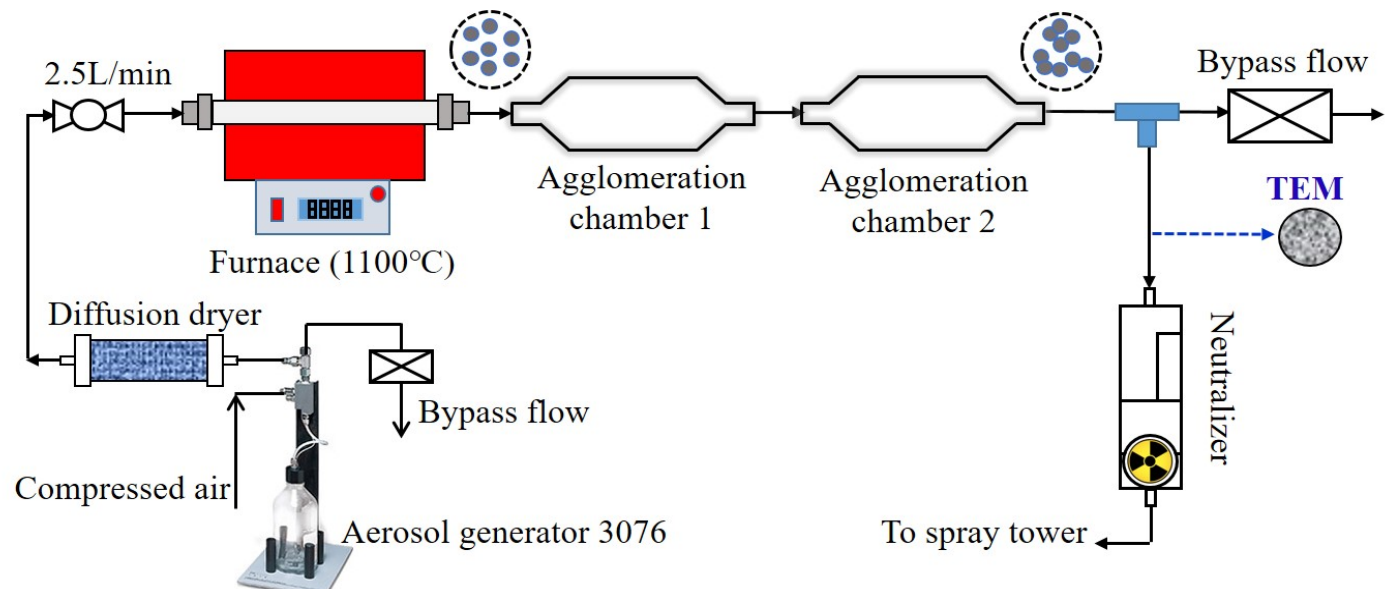

Fig. 2. Agglomerate generation system used in this study. 
Scavenging Characteristics of Hydrophilic Particles (KCl)

The performance of charged droplets to remove $\mathrm{KCl}$ particles in closed chamber was evaluated in real time for different particle mobility diameters, as shown in Fig. 3. Different scavenging stages for peak size $(120 \mathrm{~nm})$ in a continuous decay process were first studied, as shown in Fig. 3(a). In initial stage where water spray is not turned on, particle concentration in this size has no significant decrease due to wall deposition. After spraying, particle concentration was quickly reduced to $3000 \mathrm{\#} \mathrm{cm}^{-3}$ approximately for both cases, but the decline rate slowed down gradually. At that moment, a high voltage was applied on copper ring, which significantly increase the decline rate again in comparison with spraying stage. Turning off the high voltage subsequently, as shown in the second case of Fig. 3(a), the particle concentration in low level was almost indeclinable, which further proved the advantage of charged droplets to remove low-concentration particles. On this basis, $3000 \mathrm{\#} \mathrm{cm}^{-3}$ was selected as the initial concentration to underline the effect of droplet charging on particle decay rate for different mobility sizes, as shown in Figs. 3(b)-3(d). In the case of $70 \mathrm{~nm}$, wall deposition plays a non-ignorable role even in that low level due to the greater diffusion effect in this size. However, the electrical interaction between charged droplets and particles is still the dominant mechanism to collect nanoparticles. For cases of both $120 \mathrm{~nm}$ in Fig. 3(c) and $220 \mathrm{~nm}$ in Fig. 3(d), the effect of wall deposition could be neglected as a comparison with Fig. 3(b). At the stage of spraying, the decay rate of particle concentration increases with raising either spraying flowrate or high voltage but the magnitudes of increase are significantly different for two mobility sizes. In Fig. 3(c), the hydrodynamic mechanism has a marginal effect while the electrical interaction plays an extremely crucial role on collecting particles in that size. However, the effect is completely opposite for particles in $220 \mathrm{~nm}$, as shown in Fig. 3(d). The decay rate under uncharged droplets is already pretty quickly, thus the droplets charging only has a little effect on improving removal efficiency. Therefore, the biggest assistance with an addition of electric field to remove particles occurs in harsh conditions where the conventional spraying has a low efficiency.

\section{Scavenging Characteristics of Hydrophobic Particles (DEHS)}

Similar to section 3.1, real-time removal processes of DEHS particles by charged and uncharged droplets were investigated for different water flowrates and mobility sizes, as shown in Figs. 4(a) and 4(b). The effect of wall deposition on removing particles was also evaluated separately to quantify its contribution within all collection mechanisms.
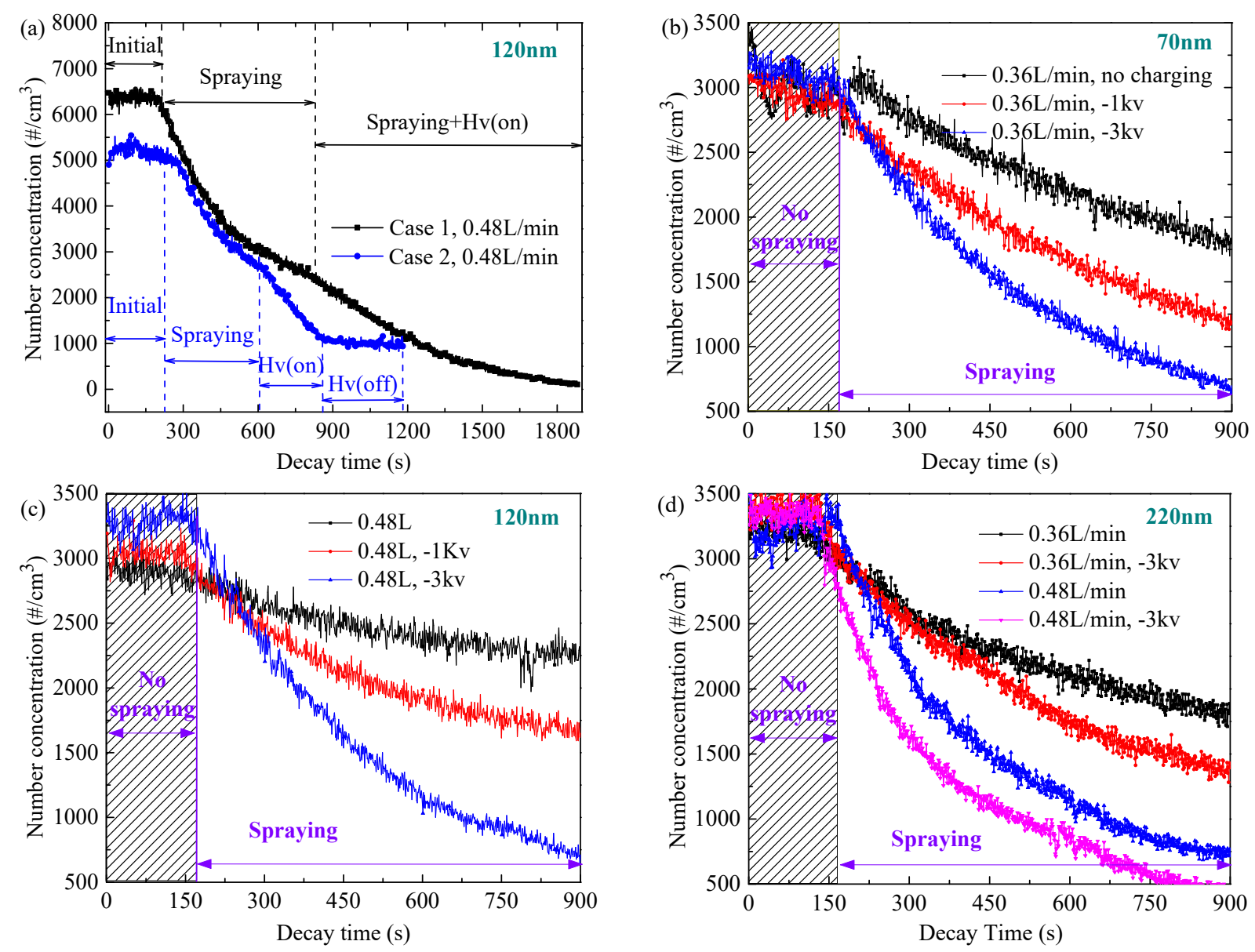

Fig. 3. Real-time decay of $\mathrm{KCl}$ particle concentration in closed chamber for different particle mobility diameters. (a) $120 \mathrm{~nm}$ under different spraying stages, (b) $120 \mathrm{~nm}$ under different voltages, (c) $70 \mathrm{~nm}$, (d) $220 \mathrm{~nm}$. 

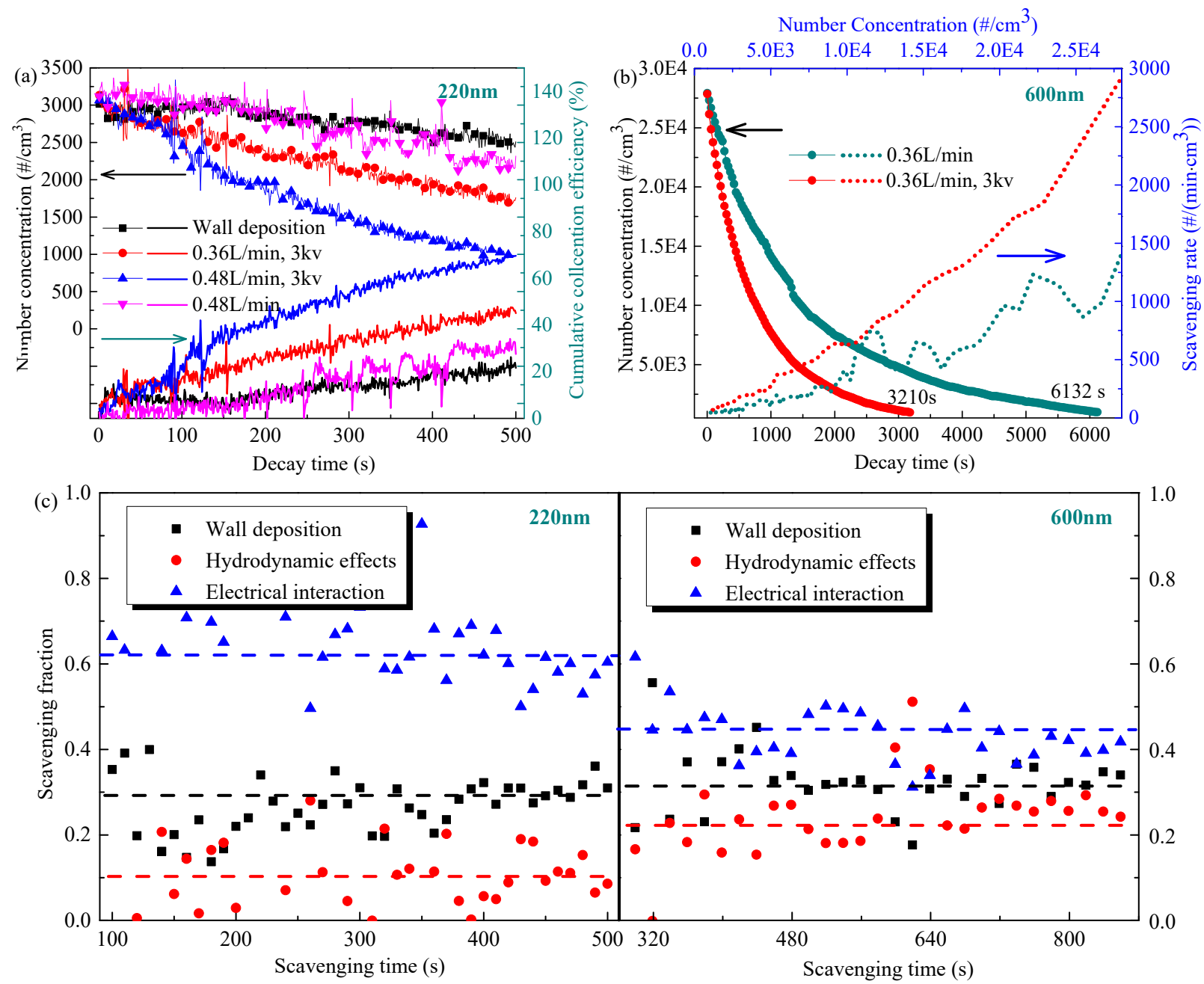

Fig. 4. Removal of DEHS particles in closed chamber. (a) scavenging process of particle in $220 \mathrm{~nm}$, (b) $600 \mathrm{~nm}$, (c) contribution fraction of different mechanisms for $220 \mathrm{~nm}$ and $600 \mathrm{~nm}$.

It can be clearly seen in Fig. 4(a) that particle concentration drops slowly in uncharged spraying, which is almost in the same magnitude as wall deposition. Once DEHS particles, however, were applied an image force by charged droplets, its decay rate has a substantial increase. Coupling with electric field, the water flowrate also plays a positive role in collecting DEHS particles, which takes effect in full range of mobility size discussed in this study. From the viewpoint of cumulative collection efficiency in Fig. 4(a), a great difference could be found that $70 \%$ and $30 \%$ after $500 \mathrm{~s}$ correspond to uncharged and charged droplets in high flowrate, respectively. Beyond that, the noise fluctuation with decay time in charged spray is smaller than that in uncharged spray, which also could be found for scavenging rate of particle in $600 \mathrm{~nm}$. As shown in Fig. 4(b), not only is scavenging time starting from same high concentration $\left(28,000 \mathrm{H} \mathrm{cm}^{-3}\right)$ reduced by one-half due to droplets charging, but also the scavenging rate keeps a more stable monotonous trend with scavenging time. A plausible reason for this phenomenon is that positive charges on each droplet have a repulsion force on other charged droplets, which disperse a great number of droplets in chamber more evenly.
In order to quantify contributions of different collection mechanisms in scavenging process, computing results for two cases, $220 \mathrm{~nm}$ and $600 \mathrm{~nm}$, were shown in Figs. 4(c) and $4(\mathrm{~d})$, respectively. First of all, thermophoretic and diffusiophoretic effects could be neglected due to almost undifferentiated temperature between droplets and particles. Only three kinds of important mechanisms, including wall deposition, electrical interaction and hydrodynamic effects, were taken into account in this study. It could be clearly seen in Fig. 4 that electrical interaction is the most critical mechanism for both cases while hydrodynamic mechanism has a marginal effect compared other two mechanisms. Besides, wall deposition contributes $30 \%$ approximately for both cases, indicating no significant difference to remove DEHS particles in different sizes. However, contribution fraction of electrical interaction is reduced from $60 \%$ to $45 \%$ while that of hydrodynamic effect increases from $10 \%$ to $22 \%$ approximately with mobility size. In this regard, inertial impaction accounts for the larger proportion in collecting particles of $600 \mathrm{~nm}$, which relatively weakens the ascendancy of electrical interaction. 


\section{Overall Comparison of Scavenging Characteristics of DEHS and KCl Particles}

Figs. 5(a) and 5(b) depict multistage concentration distributions and collection efficiencies of $\mathrm{KCl}$ particle in full size range for cases of both uncharged and charged droplets, respectively. With an increase of spraying time, particle concentration in each particle size declines gradually and fractional collection efficiency increases correspondingly for both cases. Particularly in Fig. 5(a), both the peak of inverse U-shape curve and the valley of U-shape curve occur in the same particle size, suggesting that particle concentration seems to affect collection efficiency of $\mathrm{KCl}$ particles. As a comparison, Fig. 5(b) shows a slight deviation of locations where two different extremums emerge in, which might be because droplet charging changes original trapping equilibrium by hydrodynamic effects. Besides, the minimal collection efficiency after ten minutes increases from $42 \%$ to $61 \%$ due to the addition of electrical interaction between droplets and particles.

Similarly, the variations of DEHS particle concentration and collection efficiency with mobility diameter at different spraying stages were shown in Fig. 6. As a supplement, collection efficiency in larger size by APS measurement was also depicted, as shown in Fig 7. Combining Fig. 6 with Fig. 7, the valley diameter has a large gap with peak size, stating clearly DEHS particle concentration has a marginal effect on fractional collection efficiency. Whether for Fig. 6 or Fig. 7, it could be clearly seen that droplet charging increases the minimal collection efficiency of DEHS particles from $38 \%$ to $81 \%$, indicating a completely significant increment. Even though peak size and absolute concentration of DEHS particles are slightly different from that of $\mathrm{KCl}$ particles, it could also be acquired that droplet charging take more effects on removal of hydrophobic particles.

When a hydrophobic particle migrates close to a droplet, it may adhere to the surface of droplet or rebound from droplet surface, which depends on impact angle between particle and droplet (Zuo et al., 2017). As more particles accumulate to the droplet, a dendritic structure (Sumiyoshitani et al., 1984) may occur on charged droplet surface if particles are rigid spheres, and a big ellipsoid probably corresponds to aggregated oil particles. In case of droplet charging, the electrical interaction could offer a stronger particle adhesion on droplet surface than Van der Waals force only, reducing
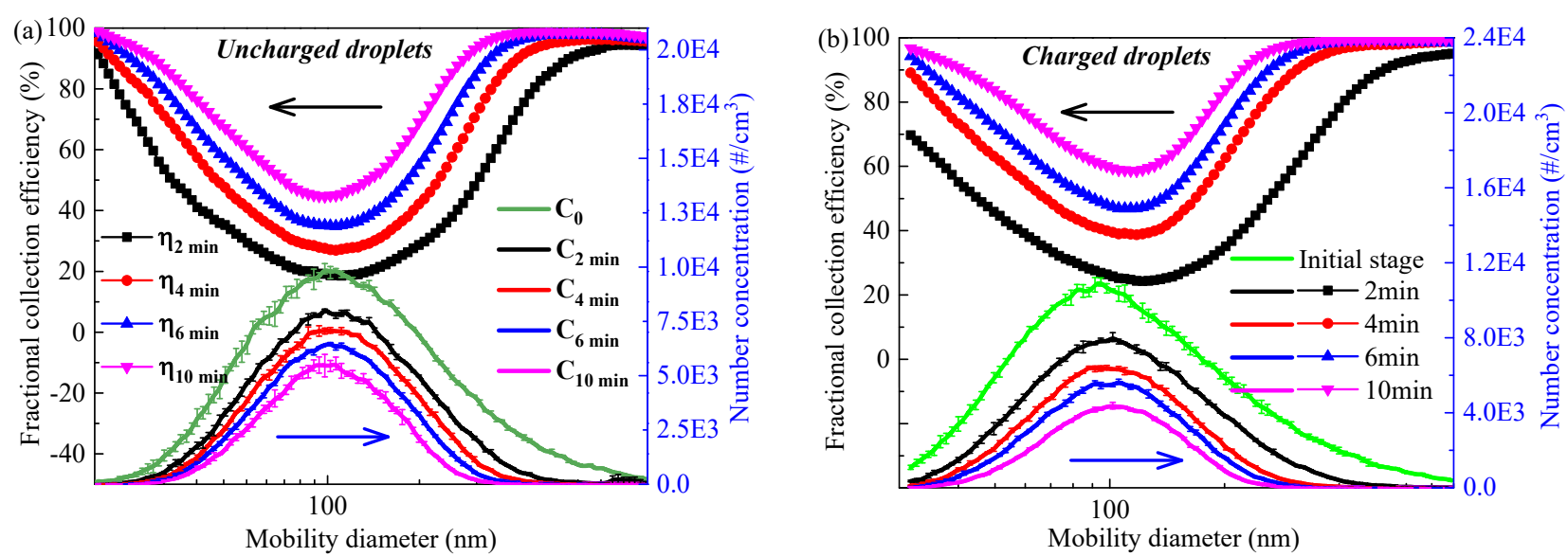

Fig. 5. Fractional collection efficiency and number concentration distribution of $\mathrm{KCl}$ particles. (a) with uncharged droplets, (b) charged droplets.
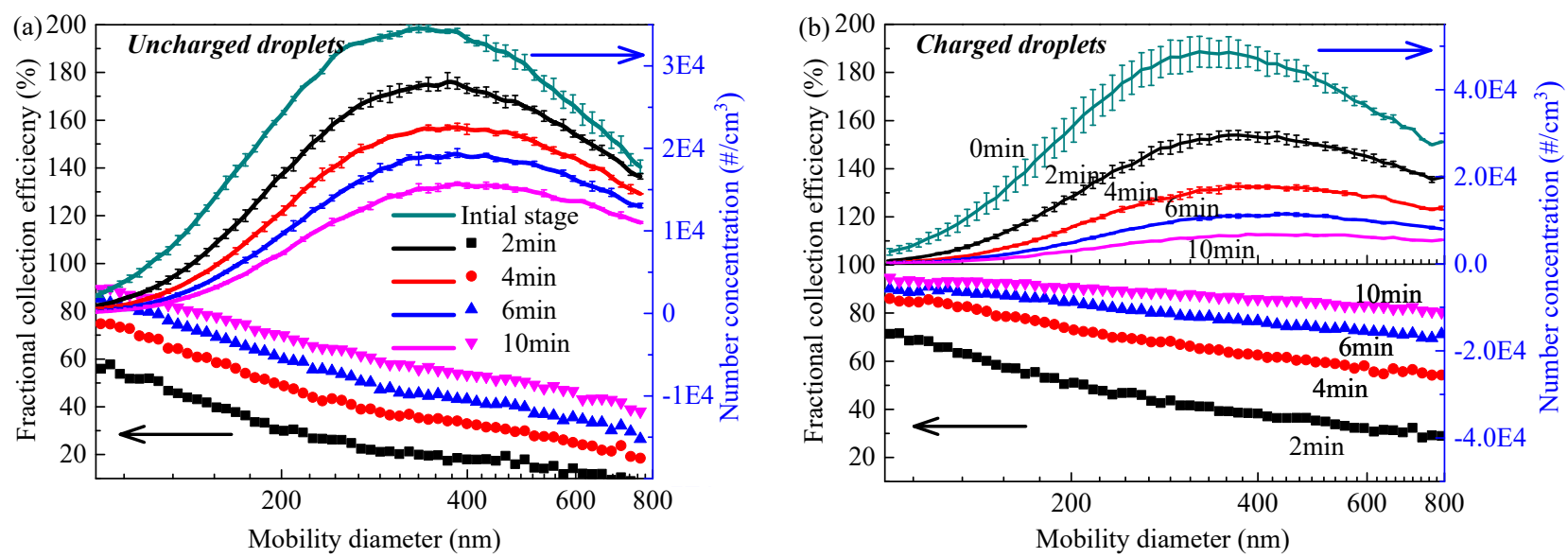

Fig. 6. Fractional collection efficiency and number concentration distribution of DEHS particles. (a) with uncharged droplets, (b) charged droplets. 


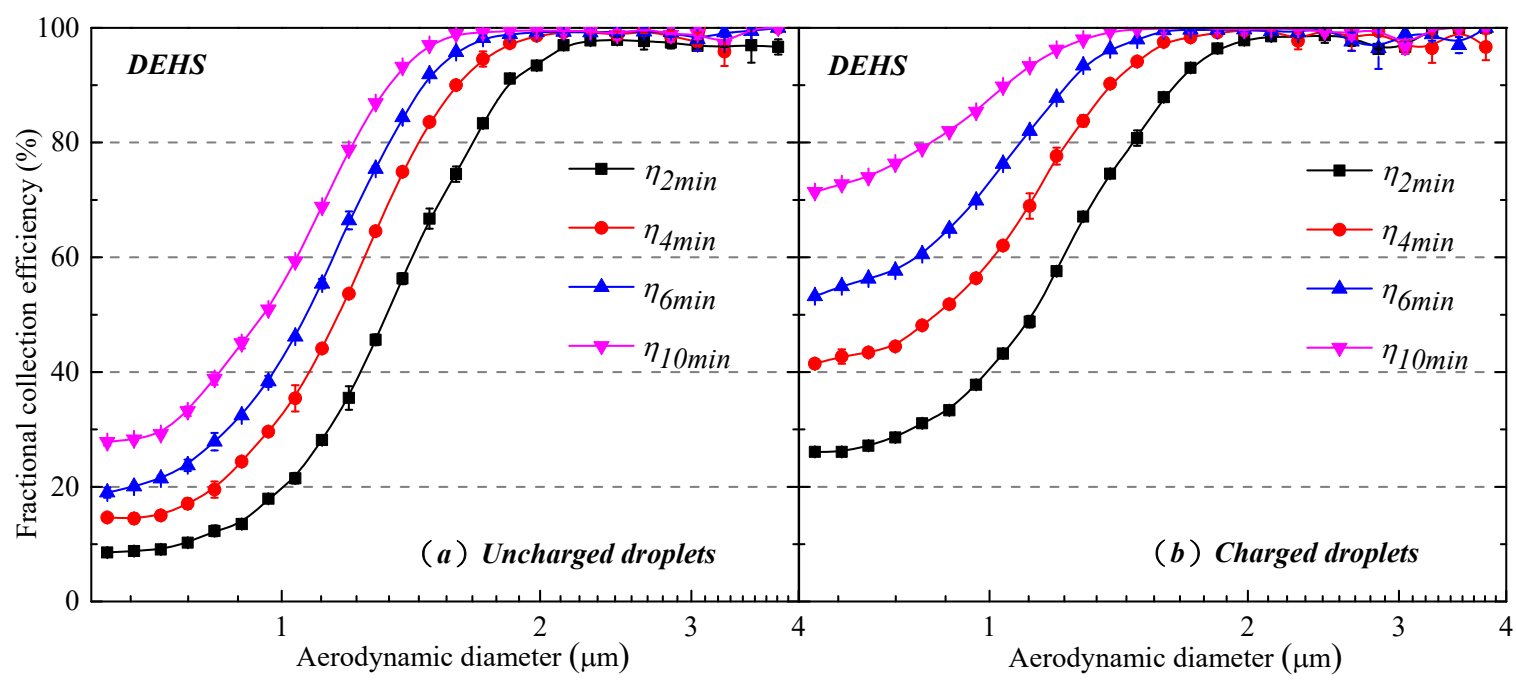

Fig. 7. Fractional collection efficiency of DEHS particles measured by APS. (a) with uncharged droplets, (b) charged droplets.

particle shedding due to droplet oscillation in settlement process. With reference to a hydrophilic particle, it could pierce droplet surface and get into the inside when colliding with a droplet (Sumiyoshitani et al., 1984). Another conceivable reason is that a hydrophilic particle may grow up due to condensation effect in a super-saturated environment (Weingartner et al., 1995; Bologa et al., 2001; Du et al., 2016), so it could be captured by a neutral droplet more easily. Therefore, net efficiency increment for capturing hydrophobic particles is relatively larger than that for collecting hydrophilic particles by charged droplets.

\section{Comparison between Agglomerates and Spherical Particles}

Two-dimensional images of single agglomerate and nearly spherical particle taken by TEM were shown in Fig. 8(a) and $8(\mathrm{~b})$, respectively. It can be clearly seen that the agglomerate consists of many primary particles and has a high aspect ratio, which significantly distinguishes from nearly spherical particle. Fig. 9 depicts concentration distribution and fractional collection efficiency of agglomerate in full size range to compare with that of spherical particle. Similarly, droplet charging plays a considerable role in improving collection efficiency of hydrophilic particles regardless of particle shape. In both scenarios, significant synergism by droplet charging occurs after running for ten minutes when particle concentration is at a low level. In order to expatiate the effect of particle morphology on its removal, the comparison of classified particles in a lower initial concentration was further shown in Fig. 10. In uncharged condition, the declining rates of concentration of both agglomerate and sphere are almost identical in early stage. Subsequently, the difference significantly emerges in both spraying flowrates, suggesting a higher removal efficiency for agglomerates in a low concentration. In the case of droplet charging, particle concentration is distinctly reduced to a lower level within the same time. Particularly, the cut-off concentration to distinguish agglomerate from sphere drops to a lower level than that in Fig. 10(a), which indicates a smaller gap caused by particle shape with an addition of electric field.

In the absence of electric field, trapping mechanisms of particles by neutral droplets mainly involves inertial impaction, Brownian diffusion and directional interception (Carotenuto et al., 2010). Similar to the theory of particles trapped by a fibrous filter (Kim et al., 2009), particle diameter used in calculating Stokes number and Schmidt number refers to mobility diameter preferentially, thus inertial impaction and Brownian diffusion play marginal effects on improving removal efficiency of agglomerates. Directional interception, however, is the crucial factor due to the importance of $H$ in Eq. (2).

$E_{d i}=4 H\left[\omega^{-1}+\left(1+2 R e^{0.5}\right) H\right]$

where $E_{d i}$ is directional interception parameter, $R e$ is droplet Reynolds number, $\omega$ is ratio of water viscosity to gas viscosity and $H$ ratio of characteristic particle dimension to droplet diameter. For spherical particle, characteristic particle dimension is geometric diameter while it refers to the maximum projected length $(L)$ for agglomerates, as shown in Eq. (3).

$H=L / D$

Obviously, $L$ of an agglomerate is greatly larger than geometric diameter of a sphere at the same mobility. Therefore, the removal of loose agglomerates is more effective than that of spheres by droplets. However, under droplets charging, a long-distance force provided by electric field is applied in collecting particles, which weakens the influence of particle morphology to some extent.

\section{CONCLUSIONS}

In this study, a home-made electrostatic scrubber was designed to investigate the effects of particle hydrophilicity and morphology on its removal. From experiments the 

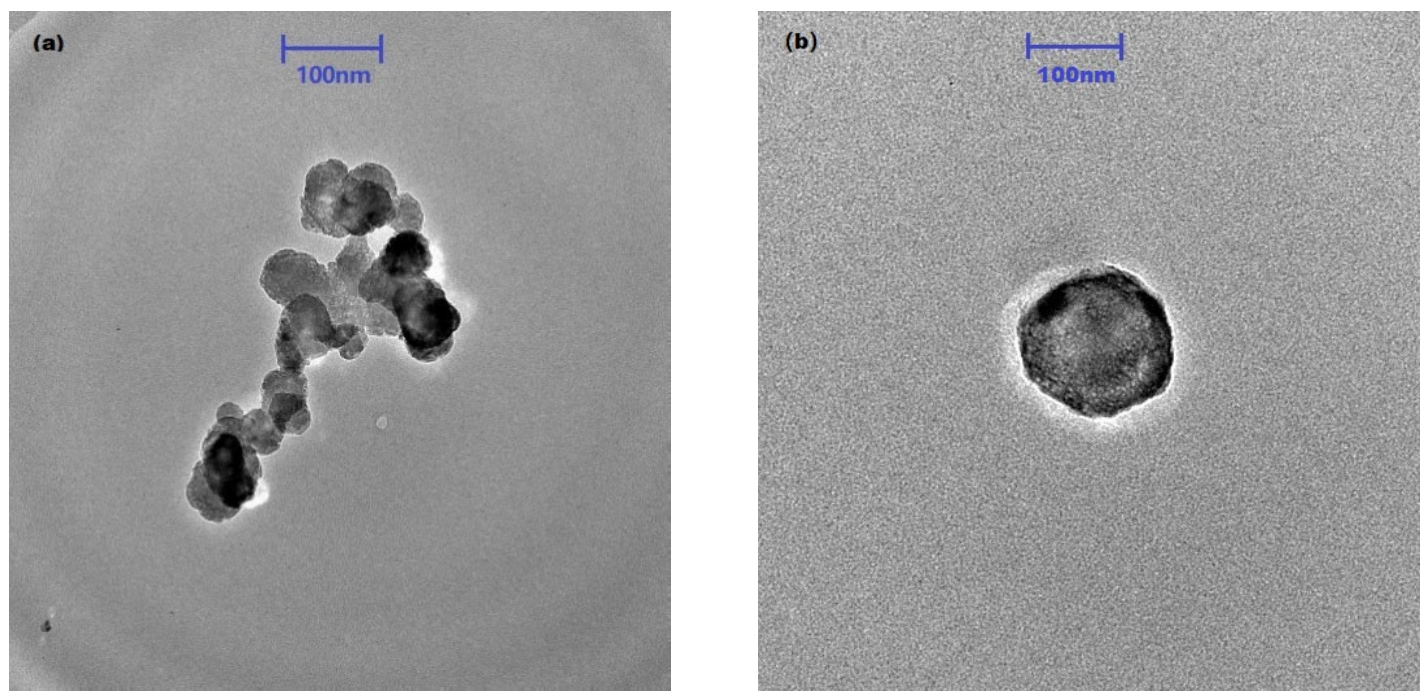

Fig. 8. Particle morphology measured by TEM. (a) $\mathrm{KCl}$ agglomerate, (b) nearly spherical $\mathrm{KCl}$ particle.
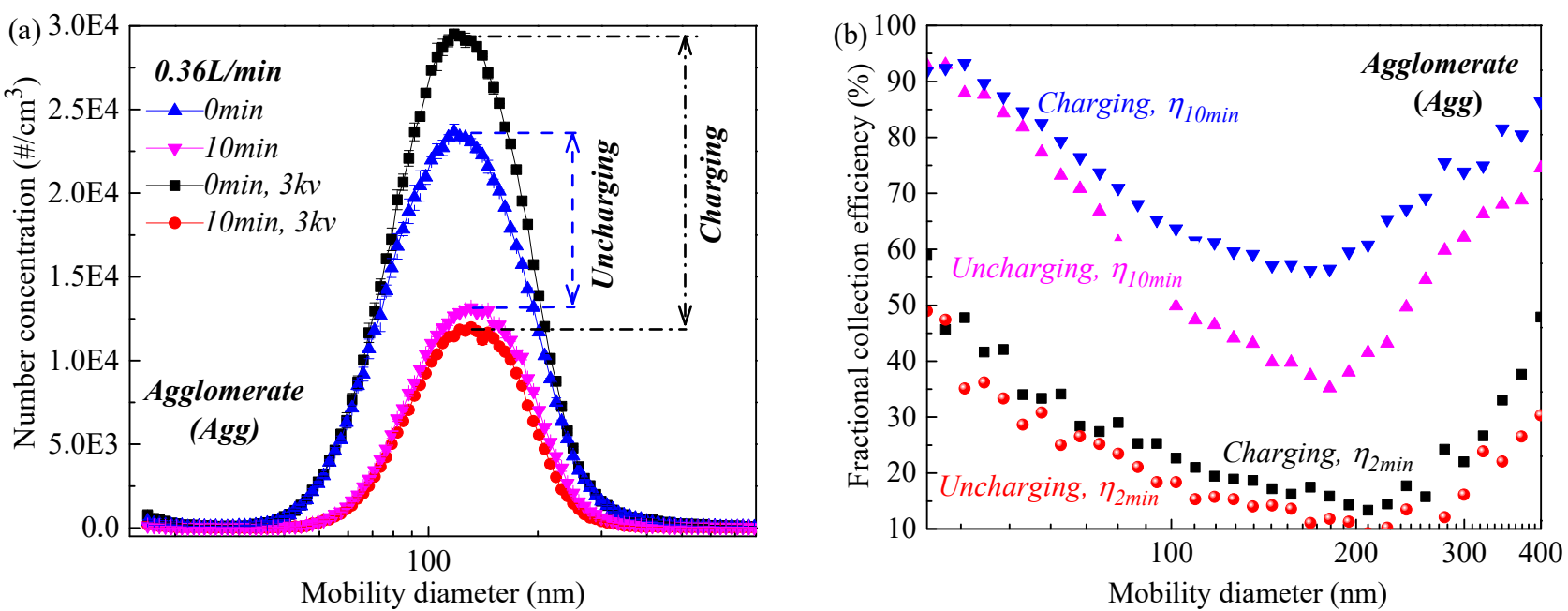

Fig. 9. $\mathrm{KCl}$ agglomerate distribution and collection efficiency. (a) number concentration distribution, (b) fractional collection efficiency.
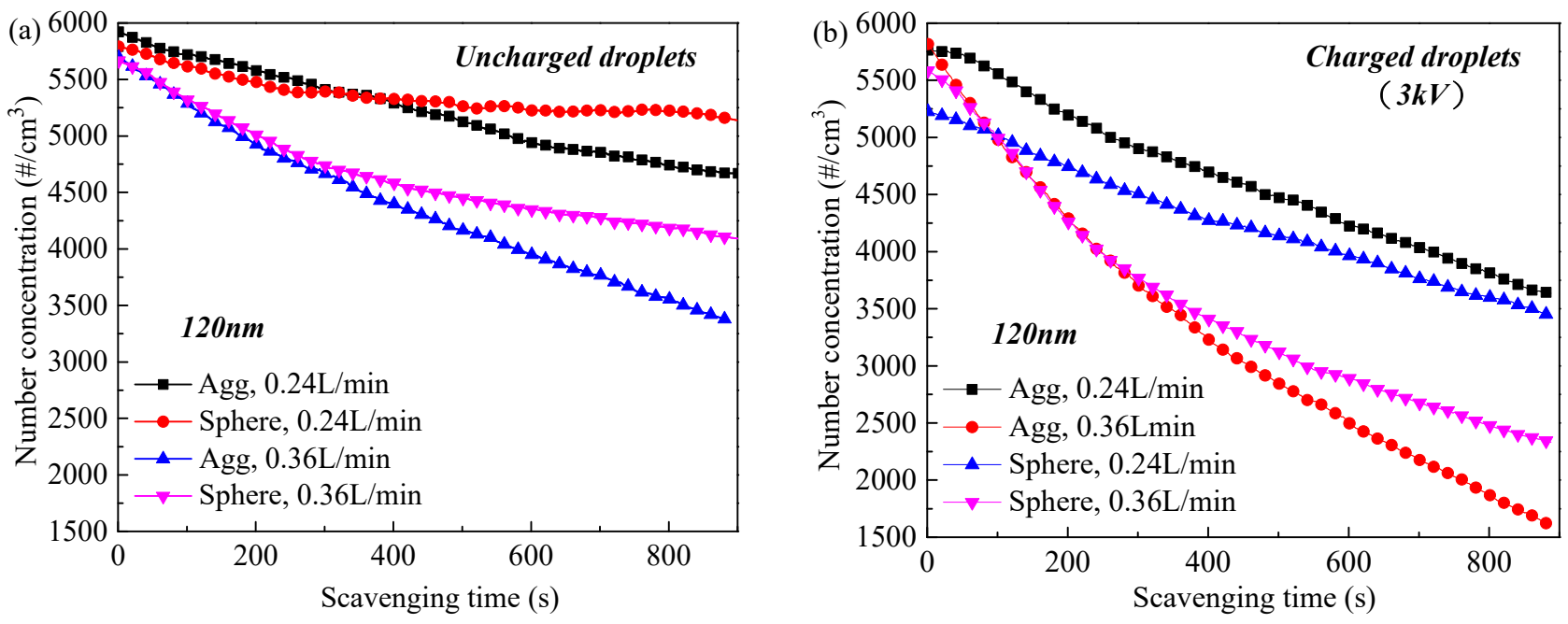

Fig. 10. Comparison between agglomerate and nearly spherical particle in $120 \mathrm{~nm}$. (a) uncharged droplets, (b) charged droplets. 
following key conclusions could be derived for particle removal by charged droplets:

(1) For trapping hydrophilic particles, biggest assistance of electrical interaction occurs in harsh conditions, such as low particle concentration and intermediate particle size, where traditional spray has a lowest efficiency. However, droplet charging enhances the operational stability of traditional spray to collect DEHS particles in all tested sizes.

(2) Compared with $\mathrm{KCl}$ particles, droplet charging plays a more crucial role in improving removal efficiency of DEHS particles, suggesting a great potential for trapping hydrophobic particles by upgrading conventional spray with minimal efforts.

(3) As a comparison with nearly spherical particles, a higher collection efficiency corresponds to loose agglomerates in a low particle concentration due to interception mechanism, but the difference could be weakened with an addition of electric field onto water spray. Follow-up studies should focus on building a swarm-particle model to predict collection efficiency of particles with different properties.

\section{ACKNOWLEDGMENTS}

The authors thank the support of members of the Center for Filtration Research: 3M Corporation, A.O. Smith Company, Applied Materials, Inc., BASF Corporation, Boeing Company, Corning Co., China Yancheng Environmental Protection Science and Technology City, Cummins Filtration Inc., Donaldson Company, Inc., Entegris, Inc., Ford Motor Company, Guangxi Wat Yuan Filtration System Co., Ltd, MSP Corporation; Samsung Electronics Co., Ltd., Xinxiang Shengda Filtration Technology Co., Ltd., TSI Inc., W. L. Gore \& Associates, Inc., Shigematsu Works Co., Ltd., and the affiliate member National Institute for Occupational Safety and Health (NIOSH). The author also thanks the project of China General Administration of Quality Supervision, Inspection and Quarantine (2017QK178) and the financial aid from the program of China Scholarship Council (CSC).

\section{REFERENCES}

Balachandran, W., Jaworek, A., Krupa, A., Kulon, J. and Lackowski, M. (2003). Efficiency of smoke removal by charged water droplets. J. Electrostat. 58: 209-220.

Becerra, T.A., Wilhelm, M., Olsen, J., Cockburn, M. and Ritz, B. (2012). Ambient air pollution and autism in Los Angeles county, California. Environ. Health Perspect. 121: 380-386.

Bologa, A., Paur, H. and Wascher, T. (2001). Electrostatic charging of aerosol as a mechanism of gas cleaning from submicron particles. Filtr. Sep. 38: 26-30.

Cao, Q., Pui, D.Y.H. and Lipiński, W. (2015). A concept of a novel solar-assisted large-scale cleaning system (SALSCS) for urban air remediation. Aerosol Air Qual. Res. 15: 1-10.

Cao, Q., Huang, M., Kuehn, T.H., Shen, L., Tao, W., Cao,
J. and Pui, D.Y.H. (2018). Urban-scale SALSCS, Part II: A parametric study of system performance. Aerosol Air Qual. Res. 18: 2879-2894.

Carotenuto, C., Di Natale, F. and Lancia, A. (2010). Wet electrostatic scrubbers for the abatement of submicronic particulate. Chem. Eng. J. 165: 35-45.

Chang, D.Q., Chen, S.C. and Pui, D.Y.H. (2016). Capture of sub-500 nm particles using residential electret HVAC filter media-experiments and modeling. Aerosol Air Qual. Res. 16: 3349-3357.

Chen, S.C., Chang, D.Q., Pei, C., Tsai, C.J. and Pui, D.Y.H. (2016). Removal Efficiency of bimodal $\mathrm{PM}_{2.5}$ and $\mathrm{PM}_{10}$ by electret respirators and mechanical engine intake filters. Aerosol Air Qual. Res. 16: 1722-1729.

Choi, H.J., Kumita, M., Hayashi, S., Yuasa, H., Kamiyama, M., Seto, T., Tsai, C.J. and Otani, Y. (2017). Filtration properties of nanofiber/microfiber mixed filter and prediction of its performance. Aerosol Air Qual. Res. 17: 1052-1062.

D’Addio, L., Di Natale, F., Carotenuto, C., Balachandran, W. and Lancia, A. (2013). A lab-scale system to study submicron particles removal in wet electrostatic scrubbers. Chem. Eng. Sci. 97: 176-185.

D'Addio, L., Carotenuto, C., Balachandran, W., Lancia, A. and Di Natale, F. (2014). Experimental analysis on the capture of submicron particles $\left(\mathrm{PM}_{0.5}\right)$ by wet electrostatic scrubbing. Chem. Eng. Sci. 106: 222-230.

Di Natale, F., Carotenuto, C., D’Addio, L., Jaworek, A., Krupa, A., Szudyga, M. and Lancia, A. (2015). Capture of fine and ultrafine particles in a wet electrostatic scrubber. J. Environ. Chem. Eng. 3: 349-356.

Di Natale, F., Carotenuto, C., D'Addio, L. and Lancia, A. (2016). Effect of gas temperature on the capture of charged particles by oppositely charged water droplets. Aerosol Sci. Technol. 50: 110-117.

Di Natale, F., Manna, L., La Motta, F., Colicchio, R., Scaglione, E. Pagliuca, C. and Salvatore, P. (2018). Capture of bacterial bioaerosol with a wet electrostatic scrubber. J. Electrostat. 93: 58-68.

Du, Q., Su, L., Dong, H., Gao, J., Zhao, Z., Lv, D. and Wu, S. (2016). The experimental study of a water-saving wet electrostatic precipitator for removing fine particles. $J$. Electrostat. 81: 42-47.

Genc, S., Zadeoglulari, Z., Fuss, S.H. and Genc, K. (2012). The adverse effects of air pollution on the nervous system. J. Toxicol. 2012: 1-23.

Ha, T.H., Nishida, O., Fujita, H. and Wataru, H. (2010). Enhancement of diesel particulate matter collection in an electrostatic water-spraying scrubber. J. Mar. Sci. Technol. 15: 271-279.

Han, T.T., Yang, L., Lee, K.B. and Mainelis, G. (2018). Design and development of a novel nanofiber nasal filter (NNF) to improve respiratory health. Aerosol Air Qual. Res. 18: 2064-2076.

Jaworek, A., Adamiak, K., Balachandran, W., Krupa, A., Castle, P. and Machowski, W. (2002). Numerical simulation of scavenging of small particles by charged droplets. Aerosol Sci. Technol. 36: 913-924.

Jaworek, A., Balachandran, W., Krupa, A., Kulon, J. and 
Lackowski, M. (2006a). Wet electroscrubbers for state of the art gas cleaning. Environ. Sci. Technol. 40: 61976207.

Jaworek, A., Balachandran, W., Lackowski, M., Kulon, J. and Krupa, A. (2006b). Multi-nozzle electrospray system for gas cleaning processes. J. Electrostat. 64: 194-202.

Jaworek, A., Krupa, A., Sobczyk, A.T., Marchewicz, A., Szudyga, M., Antes, T., Balachandran, W., Di Natale, F. and Carotenuto, C. (2013). Submicron particles removal by charged sprays. Fundamentals. J. Electrostat. 71: 345350.

Jaworek, A., Sobczyk, A.T., Krupa, A., Marchewicz, A., Czech, T. and Śliwiński, L. (2018). Hybrid electrostatic filtration systems for fly ash particles emission control. A review. Sep. Purif. Technol. 213: 283-302.

Kim, H.G., Kim, H.J., Lee, M.H. and Kim, J.H. (2014). Experimental study on the enhancement of particle removal efficiency in spray tower scrubber using electrospray. Asian J. Atmos. Environ. 8: 89-95.

Kim, S.C., Wang, J., Emery, M.S., Shin, W.G. and Pui, D.Y.H. (2009). Structural property effect of nanoparticle agglomerates on particle penetration through fibrous filter. Aerosol Sci. Technol. 43: 344-355.

Li, Q., Jiang, J., Duan, L., Deng, J., Jiang, L., Li, Z. and Hao, J. (2015). Improving the removal efficiency of elemental mercury by pre-existing aerosol particles in double dielectric barrier discharge treatments. Aerosol Air Qual. Res. 15: 1506-1513.

Liao, Z., Li, Y., Xiao, X., Wang, C., Cao, S. and Yang, Y. (2018). Electrostatic precipitation of submicron particles with an enhanced unipolar pre-charger. Aerosol Air Qual. Res. 18: 1141-1147.

Mazumder, M.K., Sims, R.A., Biris, A.S., Srirama, P.K., Saini, D., Yurteri, C.U., Trigwell, S, De, S. and Sharma, R. (2006). Twenty-first century research needs in electrostatic processes applied to industry and medicine. Chem. Eng. Sci. 61: 2192-2211.

Metzler, P., Weiß, P., Büttner, H. and Ebert, F. (1997). Electrostatic enhancement of dust separation in a nozzle scrubber. J. Electrostat. 42: 123-141.

Park, J.W., Kim, C., Park, J. and Hwang, J. (2018). Computational fluid dynamic modelling of particle charging and collection in a wire-to-plate type singlestage electrostatic precipitator. Aerosol Air Qual. Res. 18: 590-601.

Pilat, M.J., Jaasund, S.A. and Sparks, L.E. (1974). Collection of aerosol particles by electrostatic droplet spray scrubbers. Environ. Sci. Technol. 8: 360-362.

Sobolewski, M., Anderson, T., Conrad, K., Marvin, E., Klocke, C., Morris-Schaffer, K., Allen, J.L. and Cory-Slechta,
D.A. (2018). Developmental exposures to ultrafine particle air pollution reduces early testosterone levels and adult male social novelty preference: Risk for Children's sexbiased neurobehavioral disorders. NeuroToxicology 68: 203-211.

Song, C., Wu, L., Xie, Y., He, J., Chen, X., Wang, T., Lin, Y., Jin, T., Wang, A., Liu, Y., Dai, Q., Liu, B., Wang, Y. and Mao, H. (2017). Air pollution in China: Status and spatiotemporal variations. Environ. Pollut. 227: 334 347.

Sumiyoshitani, S., Okada, T., Hara, M. and Akazaki, M. (1984). Direct observation of the collection process for dust particles from an air stream by a charged water droplet. IEEE Trans. Ind. Appl. 20: 274-281.

Weingartner, E., Baltensperger, U. and Burtscher, H. (1995). Growth and structural change of combustion aerosols at high relative humidity. Environ. Sci. Technol. 29: 2982-2986.

Yu, S. (2014). Water spray geoengineering to clean air pollution for mitigating haze in China's cities. Environ. Chem. Lett. 12: 109-116.

Zhang, J., Reid, J.S., Alfaro-Contreras, R. and Xian, P. (2017). Has China been exporting less particulate air pollution over the past decade? Geophys. Res. Lett. 44: 2941-2948.

Zhang, Q., Liu, F., Yang, T.Y., Si, X.L., Hu, G.R. and Chang, C.T. (2017). Deciphering effects of surface charge on particle removal by $\mathrm{TiO}_{2}$ polyacrylonitrile nanofibers. Aerosol Air Qual. Res. 17: 1909-1916.

Zhao, H. and Zheng, C.G. (2008). Modeling of gravitational wet scrubbers with electrostatic enhancement. Chem. Eng. Technol. 31: 1824-1837.

Zheng, C., Chang, Q., Lu, Q., Yang, Z., Gao, X. and Cen, K. (2016). Developments in unipolar charging of airborne particles: Theories, simulations and measurements. Aerosol Air Qual. Res. 16: 3037-3054.

Zhou, X., Xu, Y., Yuan, S., Wu, C. and Zhang, H. (2015). Performance and potential of solar updraft tower used as an effective measure to alleviate Chinese urban haze problem. Renewable Sustainable Energy Rev. 51: 14991508.

Zuo, Z., Wang, J., Huo, Y. and Xu, R. (2017). Numerical study of particle motion near a charged collector. Particuology 32: 103-111.

Received for review, April 16, 2019 Revised, July 20, 2019 Accepted, September 13, 2019 